\title{
Tratamiento neuropsicológico de «dolor de miembro fantasma" a propósito de un caso
}

\author{
Mas Esquerdo J. ${ }^{1}$, Maruenda Fernández. R. ${ }^{2}$, Robles Sánchez JI. ${ }^{3}$
}

Sanid. mil. 2013; 69 (3): 195-202; ISSN: 1887-8571

\begin{abstract}
RESUMEN
En el presente artículo, presentamos un caso de tratamiento de dolor de miembro fantasma en una soldado de las Fuerzas Armadas Españolas, que sufrió amputación de miembro inferior derecho tras la explosión de un artefacto explosivo. La paciente refiere dolor en el muñón, así como en el dorso y la planta del pie amputado. Describe el dolor de miembro fantasma (DMF) con una intensidad de 5-6/10 en una Escala de Valoración Analógica (EVA). Se practicó el tratamiento para el DMF propuesto por Ramachandran mediante la Técnica del Espejo. Se realizaron 30 sesiones, de 20-30 minutos, midiendo la intensidad del DMF al inicio y final de cada sesión mediante EVA. Al alta, y tras 4 meses de tratamiento, la paciente informa de una intensidad de dolor inferior a 1. Se concluye la eficacia de esta técnica y el inconveniente de la posible reexperimentación del trauma a través de la imagen especular que simula al miembro amputado. Asimismo, también se constata la influencia de aspectos emocionales como factores moduladores de la experiencia dolorosa. El caso descrito pone de manifiesto el sorprendente grado de plasticidad del cerebro adulto, y la poderosa interacción de la vía visual sobre la vía sensitivo-motora. Por último, necesitamos seguir investigando en el ámbito de la asistencia sanitaria militar operativa para poder ofrecer tratamientos eficaces en este tipo de patologías, tan frecuentes en los escenarios bélicos actuales.
\end{abstract}

PALABRAS CLAVE: Neuropsicología, Dolor miembro fantasma, Terapia del espejo, Amputación.

\section{Neurpsychological treatment of phantom limb pain: A case report}

SUMMARY: In this paper we present a case of treatment of phantom limb pain suffered by a soldier of the Spanish Armed Forces, who underwent right lower-limb amputation following the explosion of an explosive device. The patient reports stump pain, as well as pain in the top and sole of the amputated foot. She describes phantom limb pain (PLP) with an intensity level of 5-6/10 on a Visual Analogue Scale (VAS). The treatment for PLP proposed by Ramachandran is given, using the Mirror Therapy. 30 sessions, of 20-30 minutes each, are carried out, measuring PLP intensity at the beginning and at the end of each session using a VAS. Upon discharge, and after 4 months of treatment, the patient reports a pain intensity score below 1 . The effectiveness of this technique is thus concluded, as well as the inconvenience of the possibility of re-experiencing the trauma through the mirror image that simulates the amputated limb. In addition, there is also evidence of the influence of emotional aspects as factors modulating the painful experience. The case described highlights the remarkable degree of plasticity of the adult brain, and the powerful interaction of the visual pathway on the sensory-motor pathway. Lastly, we need to continue further research in the field of operational military healthcare in order to provide effective treatments for this type of pathologies, which are so prevalent in the current theatres of war.

KEY WORDS: Neuropsychology, Limb phantom pain, Mirror therapy, Amputation.

\section{PRESENTACIÓN DEL CASO}

Mujer de 25 años, militar del E.T., destacada en Afganistán, que en el mes de junio de 2011 sufre amputación de pierna derecha y heridas de diversa consideración por la explosión de un artefacto al paso del vehículo del que era conductora.

Los hechos se desarrollan en el noroeste del país donde la paciente forma parte de un convoy militar. Al paso de su vehículo estalla un artefacto explosivo de gran potencia que levanta el vehículo blindado y causa heridas de diversa consideración a los cinco ocupantes del vehículo. Como resultado de la explosión

${ }^{1}$ Cap. Psicólogo. Servicio de Psicología Clínica.

${ }^{2}$ Médico Residente de $4^{\circ}$ curso. Servicio de Rehabilitación.

${ }^{3}$ Cte. Psicólogo. Servicio de Psicología Clínica.

Hospital Central de la Defensa Gómez Ulla. Madrid. España.

Dirección para correspondencia: Jaime Mas Esquerdo. C/Romero Robledo n 3, 28008 Madrid. imasesq@.fn.mde.es

Recibido: 1 de agosto de 2012

Aceptado: 20 de marzo de 2013 sufre shock hemodinámico, lesiones catastróficas con amputación de miembro inferior derecho (MID) con diversas quemaduras en el miembro residual, fractura de extremidad proximal de cúbito derecho y reacción de estrés agudo.

La paciente es evacuada al ROLE-2, se realiza estabilización hemodinámica y amputación de emergencia con desarticulación de la rodilla (Figura 1). Posteriormente es repatriada al Hospital Central de la Defensa (HCD) donde ingresa en la Unidad de Cuidados Intensivos (UCI). A su llegada la paciente presenta un nivel de amputación no funcional con pérdida de sustancia y deficiente cobertura dérmica, por lo que se realiza amputación transfemoral por encima del surco intercondíleo (Figura 2). A los cinco días de la intervención la paciente refiere dolor en el muñón y raíz del MID, así como en el dorso y la planta del pie amputado. Tras interconsulta hospitalaria es valorada por el Servicio de Psicología Clínica. Describe el dolor de miembro fantasma con las siguientes características:

- Intensidad en la Escala de Valoración Analógica (EVA): 5-6 sobre 10 . 


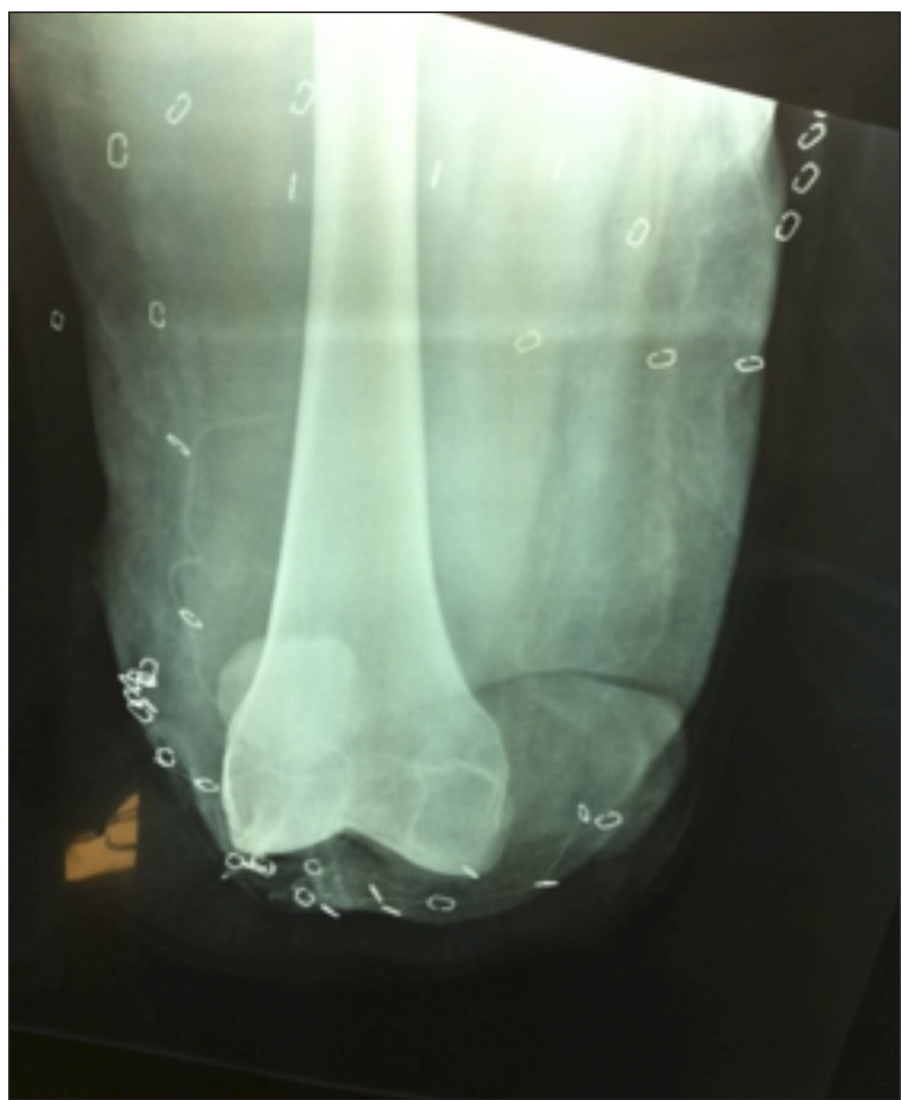

Figura 1. Imagen radiológica tras intervención en $\mathrm{ZO}$.

- Cualidad: «como una corriente eléctrica».

- Sensación: Espasmo.

- Localización: en el puente del pie.

- Vivencia: agobiante, angustioso, desesperante, constante.

En la anamnesis de la paciente no constan antecedentes psicopatológicos significativos.

La valoración e intervención por parte del Servicio de Rehabilitación se ve dificultada por los problemas dérmicos del muñón. Las múltiples quemaduras en el miembro amputado requieren sucesivas curas por lo que se retrasa la protetización unos 3 meses. Aparece un cuadro ansioso-depresivo reactivo a la amputación, que requiere soporte farmacológico y psicológico.

Por parte del Servicio de Rehabilitación se realizan ejercicios de potenciación muscular de miembros superiores e inferiores, con potenciación de la musculatura pelvitrocantérea (para evitar el flexo de MID) y tratamiento electroterápico y manual del muñón. Finalmente comienza la protetización con prótesis exoesquelética, de encaje total, con rodilla C-leg y pie acumulador de energía $\mathrm{C}$-walk, y se continúa entrenamiento y deambulación con prótesis hasta el día del alta hospitalaria unos 6 meses después de su ingreso.

\section{INTRODUCCIÓN AL CONCEPTO: DOLOR, MIEMBRO FANTASMA, DOLOR DE MIEMBRO FANTASMA}

Ambroise Paré, cirujano militar francés, fue quien describió por primera vez el dolor de miembro fantasma. Sin embargo, fue

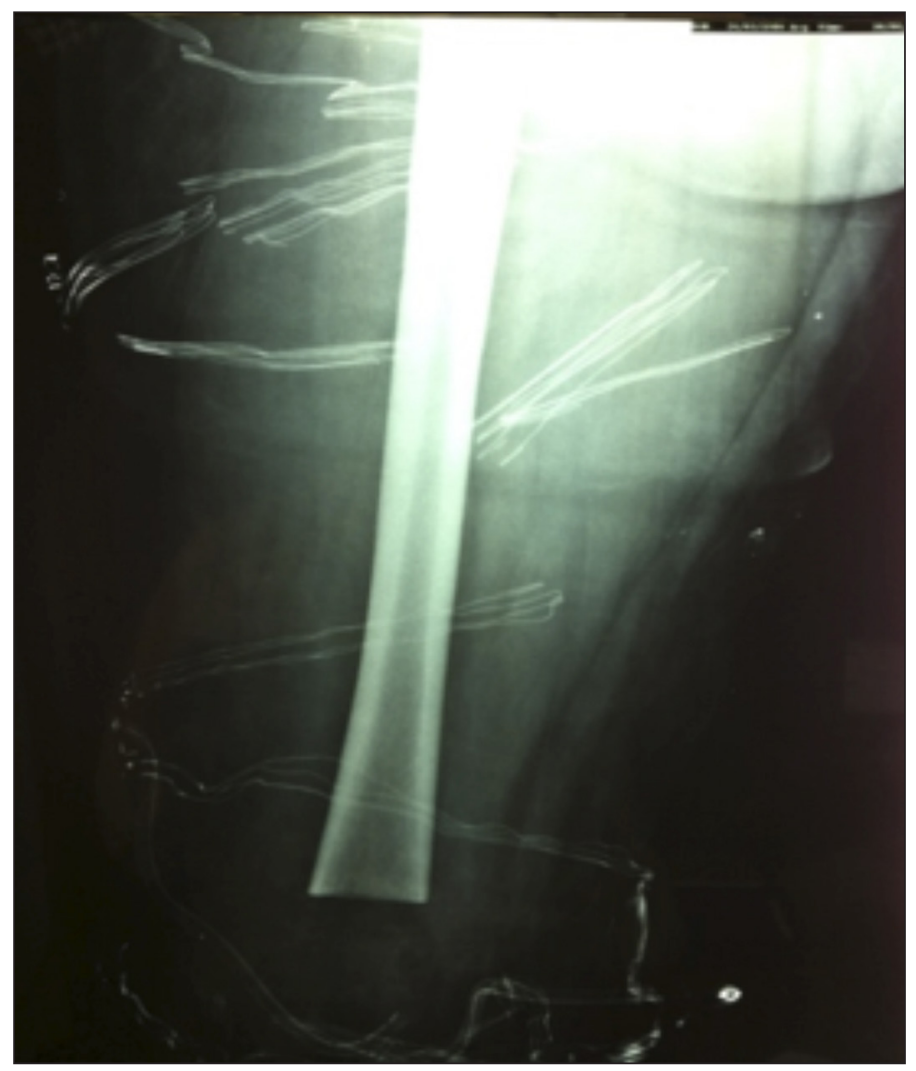

Figura 2. Imagen radiológica tras su intervención en el Hospital Central de la Defensa Gómez Ulla.

Silas Weir Mitchell, neurólogo norteamericano, quien acuñó el término «miembro fantasma» (MF) para describir las sensaciones que referían los heridos sometidos a amputaciones de extremidades en la Guerra de Secesión de los Estados Unidos de América ${ }^{1}$.

Actualmente, la clasificación de las sensaciones que aparecen tras la amputación de una extremidad ${ }^{2}$, distingue entre:

- Dolor en el muñón: La sensación dolorosa localizada en el muñón que persiste más allá de periodo de cicatrización. Esta se cree que es debido a problemas estructurales del muñón y/o su prótesis, o a dolor por la desaferentización del nervio. En este caso el dolor se localiza en el muñón mismo.

- Sensación de miembro fantasma: Las sensaciones no dolorosas percibidas en una extremidad después de que ésta ha sido amputada. La propiedad más sobresaliente de miembro fantasma es el hormigueo ${ }^{2}$.

- Dolor de miembro fantasma: La sensación dolorosa localizada en una extremidad después de que ésta ha sido amputada. Esta no corresponde al dolor localizado en el muñón ni al dolor de la cicatriz.

Aunque los datos varían, la sensación de miembro fantasma aparece entre el 70\%-100\% de los amputados, y el dolor de miembro fantasma entre el $60 \%-85 \%$ de estos casos ${ }^{3-8}$.

Se ha demostrado la relación directa de su incidencia con distintos parámetros como son: la severidad de la lesión inicial, la duración e intensidad del dolor previos a la amputación, la edad del paciente, siendo muy poco prevalente en los niños menores 
de 6 años ${ }^{1,9}$. En el postoperatorio es difícil decir si un paciente presenta dolor postoperatorio, dolor del muñón o el inicio temprano de dolor de miembro fantasma ${ }^{1,10}$.

Aunque el origen es orgánico ${ }^{11}$, los factores psicológicos pueden exacerbar el dolor, de hecho se sabe que la percepción de la intensidad del dolor está estrechamente relacionada con los estados emocionales y niveles de ansiedad experimentados por el paciente a lo largo de su convalecencia ${ }^{1,12,13}$, y recíprocamente, el dolor crónico parece modificar la personalidad de los pacientes $^{14}$.

Aunque se ha barajado la hipótesis de la existencia de factores psicopatológicos como predisponentes al DMF, diversos autores defienden que los amputados con DMF no son más neuróticos que la población general ${ }^{10,14}$.

Por último, también se informa de la reducción del DMF tras la implantación temprana de una prótesis $^{3}$, sugiriendo que el contacto con esta produciría un mayor feedback del sistema propioceptivo.

\section{FISIOPATOLOGÍA DEL DOLOR}

Las sensaciones dolorosas son recogidas en los nociceptores de la piel, que conducen dicha información a través de fibras nerviosas $\mathrm{A} \delta$ y $\mathrm{C}$, que acceden a la Sustancia Gelatinosa de Rolando (SGR) del asta posterior de la médula espinal. Es en esta donde realizan las sinapsis con la segunda neurona que, cruzando hasta el haz espinotalámico lateral contralateral, ascienden realizando múltiples sinapsis en el tálamo, hasta la corteza cerebral somatosensorial, situada en el giro postcentral del lóbulo parietal.

La información sensitiva de cada región anatómica es transmitida a un área concreta de la corteza somatosensorial. La activación repetida de cada área acaba generando una memoria somatosensorial, según el esquema propuesto por Penfield ${ }^{15}$ (Figura 3).

\section{FISIOPATOLOGÍA DEL DOLOR DEL MIEMBRO FANTASMA}

Los mecanismos subyacentes al DMF no se conocen en su totalidad pese a la amplia investigación desarrollada en esta área ${ }^{16,17}$. Las investigaciones señalan que son varios los factores implicados en la generación del DMF. Por lo general, se cree que el fenómeno se inicia por los cambios que surgen en la periferia y que alteran las entradas aferenciales que se reciben en la médula espinal y en el cerebro. Con bastante probabilidad, este se inicia periféricamente para después originarse una cascada de acontecimientos que se dirigen hacia estructuras más centrales y acaban implicando las estructuras cerebrales corticales. Esto provocaría una reorganización central y cambios que contribuyen al desarrollo del dolor fantasma. Las estructuras cerebrales pueden ser las responsables de las vívidas y complejas sensaciones que refieren algunas de las personas aquejadas de este dolor.

El desarrollo de modelos animales que imitan el dolor neuropático y las investigaciones en otras condiciones sobre el dolor neuropático, han contribuido de forma significativa a la comprensión del DMF. La lesión del nervio se sigue por una serie

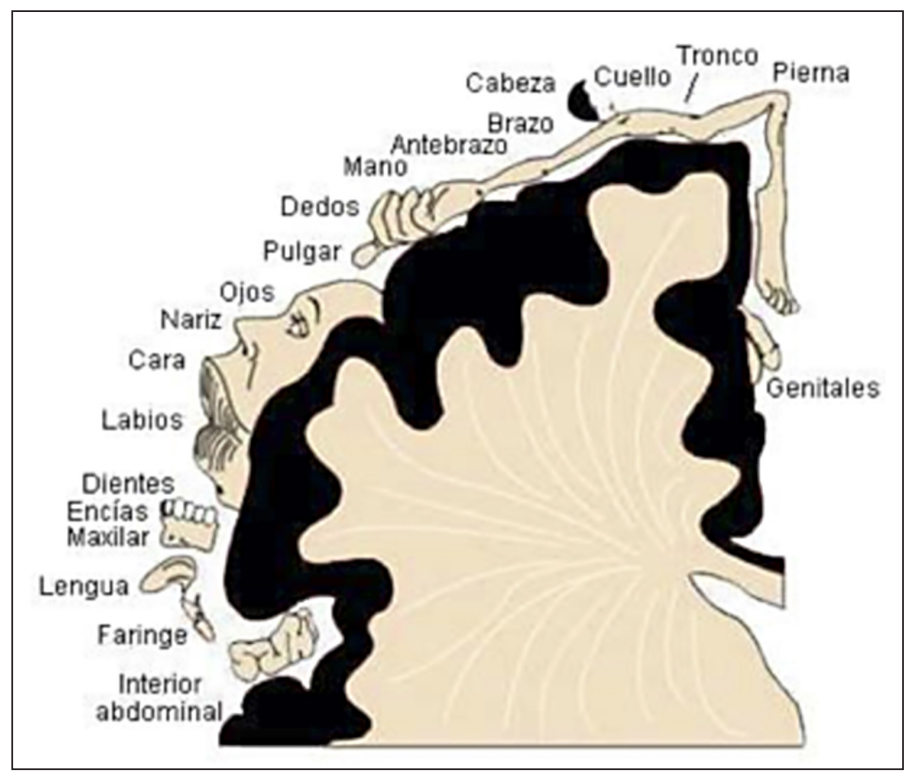

Figura 3. Esquema propuesto por Penfield ${ }^{15}$.

de cambios morfológicos, fisiológicos y químicos en los sistemas nerviosos periférico y central, y es probable que estos cambios tengan su papel en la inducción y el mantenimiento del $\mathrm{DMF}^{18}$.

\section{Factores periféricos}

Son varios los estudios clínicos que apoyan la hipótesis de que los mecanismos periféricos (sea en el muñón o en las partes centrales de las vías aferentes seccionadas), juegan un papel importante en el concepto del miembro fantasma. El DMF es significativamente más frecuente en aquellos amputados que llevan mucho tiempo con dolor que en aquellos otros sin dolor persistente $^{19}$.

Tras un corte del nervio, se aprecia universalmente la formación de neuromas. Una característica frecuente asociada a la patología del muñón es la alteración de la sensibilidad en el mismo. En el periodo inmediatamente después de la amputación, la relación existente entre el dolor fantasma y los umbrales de dolor a la presión en el muñón son inversamente proporciona$1 \mathrm{es}^{20}$. Las sensaciones fantasmas pueden modularse con diversas manipulaciones sobre el muñón ${ }^{21}$. El golpeteo de los neuromas puede incrementar el dolor fantasma. Tras la anestesia local del muñón, se pueden abolir de forma temporal las sensaciones del miembro fantasma. Los cambios en el flujo sanguíneo, también pueden alterar la percepción del miembro fantasma.

Los neuromas muestran actividad evocada espontánea y anormal tras la estimulación mecánica o química. La actividad ectópica y el aumento espontáneo y provocado desde la periferia se asume que es el resultado de un aumento de la expresión de los canales de sodio. Tras la sección completa del nervio, también ocurren estos cambios en las células de los ganglios de las raíces dorsales (GRD). Los cuerpos celulares en los GRD muestran una actividad espontánea anormal y un aumento de la sensibilidad a la estimulación mecánica y neuroquímica. El sistema nervioso simpático puede jugar también un importante papel. De los estudios con animales se sabe que la aplicación de 
noradrenalina al muñón o la activación de las fibras simpáticas postganglionares, excita y sensibiliza a las fibras nerviosas dañadas pero no a las normales.

Las alteraciones en la inervación aferente al sistema nervioso central pueden surgir como resultado de:

- La descarga ectópica de los nervios aferentes en el lugar de la amputación y de todos los neuromas resultantes de los nervios dañados (esta descarga espontánea se ha ligado a una regulación al alza del voltaje de los canales de sodio en los nervios afectados);

- El aumento de la sensibilidad de los neuromas a los estímulos mecánicos y químicos;

- La descarga ectópica de las células de los ganglios de la raíz dorsal, ligados a la regulación al alza del voltaje de los canales de sodio;

- La entrada aferente mantenida a nivel simpático desde el lugar de la amputación, secundaria al emparejamiento entre el sistema simpático y el sistema nervioso sensorial, similar al mecanismo que ocurre en el síndrome del dolor regional complejo.

\section{Factores espinales}

Las observaciones clínicas muestran que en la generación del DMF pueden estar implicados los factores medulares. Por ejemplo, el DMF puede aparecer o desaparecer después de una neoplasia en la médula espinal ${ }^{22}$. En informes de casos se ha sugerido que la analgesia espinal puede provocar dolor del miembro fantasma y que la analgesia epidural debería estar contraindicada en los amputados. Sin embargo, en un estudio prospectivo de 17 pacientes con amputación previa del miembro inferior con 23 anestésicos epidurales, solo uno desarrolló el dolor del miembro fantasma ${ }^{23}$.

Se piensa que el incremento de estimulación desde los neuromas y desde las células GRD inducen cambios a largo plazo en las neuronas de proyección central en el cuerno dorsal, incluida la actividad neuronal espontánea, la inducción de genes tempranos inmediatos, el incremento de la actividad metabólica espinal y la expansión de los campos receptivos.

También puede estar presente otro tipo de reorganización y contribuir a la sensibilización central. La substancia P se expresa normalmente en pequeñas fibras aferentes que siguen a la lesión del nervio, la substancia $P$ se puede expresar en las grandes fibras $A B$. Este interruptor fenotípico de las grandes fibras $A B$ en las fibras nerviosas nocioceptivas puede ser una de las razones del porqué los estímulos no nocivos pueden ser percibidos como dolorosos. La farmacología de la sensibilización espinal incluye un aumento de la actividad en los sistemas operados por el N-metilD-aspartato (NMDA), y muchos aspectos de la sensibilización central pueden reducirse por los antagonistas de los receptores NMDA. En los amputados humanos, el dolor evocado del muñón o dolor fantasma causado por la estimulación repetida del muñón puede reducirse por la ketamina, antagonista NMDA ${ }^{24}$.

Se han propuesto dos importantes mecanismos medulares para explicar el DMF:

- La reorganización anatómica que ocurre en la médula espinal después de la lesión periférica del nervio. Es decir, las fibras C desmielinizadas implicadas en la conducción del dolor, normalmente hacen sinapsis en la lámina 1 y 2 del cuerno dorsal. La lesión periférica del nervio puede conducir a la degeneración de estas fibras C desmielinizadas. Las grandes fibras mielinizadas $A B$, que están normalmente implicadas en el tacto, presión y propiocepción brotan de las conexiones de las láminas 3 y 4, dónde normalmente sinapsan, en la lámina 1 y 2 , que estaban previamente ocupadas por las fibras C. Este brote dentro de las láminas superficiales puede contribuir al desarrollo del dolor del miembro fantasma, de este modo el estímulo que previamente no era doloroso puede experimentarse como doloroso.

- La sensibilización central de las células dorsales del cuerno que sucede en respuesta al aumento del aluvión de estímulos dolorosos sobre el lugar de la amputación. Este estado de hiperexcitabilidad lleva al desarrollo de hiperalgesia, experimentando el paciente una exagerada respuesta a los estímulos nocivos. Los amino ácidos excitadores como el ácido glutámico y el ácido aspártico pueden estar implicados en este proceso de sensibilización, actuando a través de los receptores N-metil D-aspartato (NMDA). Pueden estar implicados otros receptores y neurotransmisorers como la sustancia $\mathrm{P}$ y el péptido relacionado con el gen de la calcitonina.

\section{Factores supraespinales}

Si la amputación produce una cascada de eventos en la periferia y en la médula espinal, es razonable asumir que estos cambios se desplazarán, eventualmente, hacia estructuras más centrales y alterarán la actividad neuronal en las estructuras corticales y subcorticales. También el concepto de miembro fantasma, con sus complejas cualidades perceptivas y sus posibilidades de modificación por varios estímulos internos (la atención, la distracción o el estrés), muestran que la imagen fantasma es un producto del cerebro.

Los estudios realizados con magnetoelectroencefalografía, en humanos y primates, han demostrado la plasticidad funcional de la corteza somatosensorial primaria tras la amputación de miembros, al producirse la reorganización cortical. En los amputados de miembros superiores, el área de la corteza somatosensorial correspondiente a los miembros desaparecidos parece recibir información sensorial de otras áreas del cuerpo que se sinapsan con las áreas adyacentes de la corteza somatosensorial. El homúnculo de Penfield muestra que el área de la cara bordea el área de la mano. Con frecuencia, los amputados de los miembros superiores, cuando se golpean en el rostro, experimentan una sensación simultánea de tacto en la cara y sobre los dedos amputados. La velocidad con la que se suceden estos cambios tras la amputación sugiere que esta reorganización probablemente es el resultado del desenmascaramiento de sinapsis ocultas en la corteza somatosensorial, en lugar de cambios directamente anatómicos. El dolor del miembro fantasma puede surgir de los errores que ocurren en este proceso de «remapeo» cortical, conduciendo a la sobreamplificación del dolor experimentado. También puede 
haber errores en las modalidades señoriales, experimentando el tacto como dolor.

En estudios con primates, tras la rizotomía dorsal, se puede demostrar una disminución del umbral a la actividad evocada en el tálamo y en la corteza, y los monos adultos muestran reorganización cortical en la que la boca y la barbilla invaden las zonas correspondientes a la representación de los brazos y los dedos que han perdido su entrada aferente normal ${ }^{25,26}$.

Los estudios en humanos también han documentado la reorganización cortical tras la amputación, utilizando diferentes técnicas de imaginería cerebral. En una serie de estudios, Flor et al. ${ }^{27,28}$ mostraron correlación entre el dolor fantasma y la cantidad de reorganización en la corteza somatosensorial. Birbaumer et al. ${ }^{29}$ estudiaron el efecto de la anestesia regional sobre la reorganización cortical en los amputados de miembros superiores y encontraron que el blocaje del plexo braquial, abolía el dolor y la reorganización en tres de seis amputados. Huse et al..$^{30}$ mostraron en un pequeño grupo de amputados que, durante el tratamiento con morfina, se redujo el dolor y la reorganización cortical.

Los cambios son también observables a niveles más subcorticales. Usando técnicas de registro y estimulación neuronal, las neuronas talámicas que normalmente no responden a la estimulación en los amputados comienzan a responder y a mostrar mapas somatotópicos ampliados ${ }^{31}$. Además de la plasticidad funcional, también se producen alteraciones estructurales tras la amputación. Draganski et al. ${ }^{32}$ han demostrado una disminución de la sustancia gris en el tálamo en 28 amputados. La disminución se relacionaba con espacio de tiempo tras la amputación y se explicó como un correlato estructural con la pérdida de las entradas aferenciales.

Por último citamos la propuesta de Ramachandran et al. ${ }^{33}$ según la cual, la génesis del miembro fantasma se debe a la reorganización cortical de las áreas adyacentes a la representación del sitio desaferentado. En efecto, estos autores observaron que la estimulación de las áreas distantes en la somatotopía, pero cercanas en la representación cortical del homúnculo, puede activar el sitio cortical de la región desaferentada, y evocar las sensaciones del miembro fantasma. La representación corporal en la corteza somatosensorial obtenida por estimulación eléctrica muestra la cercanía de las siguientes áreas: mejilla-mano, genitales-pie, cuello-mamas (Figura 3).

\section{TRATAMIENTOS DEL DOLOR}

\section{Tratamiento farmacológico para el dolor del miembro fantasma}

En el metanálisis realizado por Alviar et al. ${ }^{34}$ sobre una revisión de 583 referencias/publicaciones, seleccionan 13 estudios que implican a un total de 255 participantes. Revisaron seis grupos de medicaciones, los antagonistas de los receptores NMDA, antidepresivos, anticonvulsionantes, anestésicos, opioides y calcitonina. Diez de los estudios eran de alta calidad, y los otros tres de calidad moderada, basados en los criterios de Jadad y Van Tulder. A causa de la amplia heterogeneidad en las intervenciones farmacéuticas, el resultado de las medidas, los análisis, el informe de los resultados, la duración del seguimiento y los diseños, no fue posible agrupar los resultados para la mayoría de las intervenciones. La morfina (administrada por vía oral o intravenosa) era efectiva en la disminución de la intensidad del dolor a corto plazo, pero se informaron de efectos secundarios: estreñimiento, sedación, cansancio, mareos, sudoración, dificultad para orinar, vértigo, prurito y problemas respiratorios. Los antagonistas de los receptores NMDA, la ketamina y dextrometorfan, tenían efectos analgésicos. Los efectos adversos de la ketamina fueron más graves e incluían la pérdida de conciencia, sedación, alucinaciones y embriaguez. Los resultados de la gabapentina, en términos de alivio del dolor fueron conflictivos, pero combinando los resultados mostraban una tendencia al beneficio. La gapentina, sin embargo, no mejoraba la funcionalidad, la puntuación en depresión o la calidad del sueño, Los efectos colaterales experimentados fueron somnolencia, mareos, cefaleas y náuseas. La amitriptilina no fue efectiva en el tratamiento del DMF, con efectos colaterales de boca seca y mareos, según refiere uno de los estudios consultados. Los hallazgos para la calcitonina y los anestésicos fueron variables. Los efectos adversos de la calcitonina fueron cefaleas, vértigo, modorra, náuseas, vómitos y oleadas de calor y frío. La mayoría de los estudios estaban limitados por el pequeño tamaño de sus muestras.

Los autores, concluyen su investigación refiriendo que la efectividad a largo plazo de los opioides, antagonistas de los receptores NMDA, anticonvulsivos, antidepresivos, calcitonina y anestésicos para resultados clínicamente relevantes que incluían el dolor, funcionalidad, estado de ánimo, sueño, calidad de vida, satisfacción y efectos adversos no quedan muy claros. Morfina, gabapentina y ketamina, demostraron tendencia a la eficacia analgésica a corto plazo. Memantina y amitriptilina fueron ineficaces para el tratamiento del DMF. Los resultados, sin embargo, tienen que interpretarse con precaución puesto que se basan la mayoría de ellos en un pequeño número de estudios con tamaño muestral limitado, que además varía considerablemente y también carece de resultados sobre la eficacia y seguridad a largo plazo. La eficacia de la calcitonina, los anestésicos y el dextrametorfan, necesitan más investigación. Son necesarios ensayos aleatorios más amplios y rigurosos para hacer recomendaciones más poderosas sobre qué medicaciones pudieran ser útiles para la práctica clínica

\section{Tratamiento Rehabilitador}

- Potenciación Muscular: Debe realizarse potenciación de miembros sanos y miembro residual, y reeducación para reanudar las actividades de la vida diaria lo más precozmente posible.

- Higiene Postural: También destinada a evitar el flexo de cadera derecha.

- Electroterapia Analgésica: Para aliviar dolor del miembro residual.

- Masajes: Destinados al despegamiento cicatricial y a reducir la tensión muscular. Está demostrado que la estimulación táctil del muñón reduce la aparición de miembro fantasma.

- Protetización y estimulación táctil: Cuándo ésta sea posible, ha de realizarse lo más precozmente posible 2 . 


\section{Tratamiento Neuropsicológico}

Hay varias técnicas psicológicas que han sido descritas como útiles en el tratamiento de pacientes amputados con DMF que no presentaban alteraciones psiquiátricas relevantes. Entre estas técnicas tenemos el biofeedback y la hipnosis. Cuando predominan los factores periféricos el biofeedback parece ser una alternativa eficaz. En efecto, Sherman ${ }^{4}$ ha referido un alivio considerable del dolor de miembro fantasma para distintos tipos de dolor: biofeedback de tensión muscular para el dolor espasmódico y biofeedback de temperatura para el dolor ardiente. Técnicas como la Desensibilización y Reprocesamiento por Movimientos Oculares (DRMO) de Shapiro y la Técnica del espejo de Ramachandran también han arrojado resultados muy satisfactorios ${ }^{35-37}$.

El tratamiento neuropsicológico seguido con nuestra paciente, es el Tratamiento del dolor de miembro fantasma mediante la Técnica del Espejo propuesto por Ramachandran ${ }^{36}$.

Este procedimiento consistió en colocar un espejo en paralelo al miembro contrario al amputado. Al reflejarse en él, lo izquierdo se vuelve derecho y viceversa. Los miembros quedan entonces reflejados en una postura especular, simétrica e invertida. Es decir la persona ve reflejada en el espejo la imagen especular de sí misma, en donde su pierna derecha es la izquierda y viceversa. (Figura 4)

Como muy bien describen Solvey y Ferrazzano ${ }^{35}$, «el ejercicio consiste en colocar el miembro sano exactamente en la misma postura simétrica y especular, que ocuparía el miembro amputado. Esto quiere decir que si el miembro fantasma estuviera en pronación, contracturado o extendido, por ejemplo, el miembro sano deberá colocarse exactamente en la misma postura. Al observar el paciente su imagen en el espejo, el reflejo de su miembro sano estará ocupando visualmente el lugar de la localización del inexistente, de modo que tiene la «ilusión óptica» de que el fantasma se ha regenerado. Si el miembro perdido es el izquierdo, el paciente «ve» en el espejo el miembro izquierdo completo. Se deben realizar luego movimientos con el miembro sano, y mirándose al espejo el paciente recibirá la retroalimentación visual de que el miembro fantasma es el que se está moviendo».

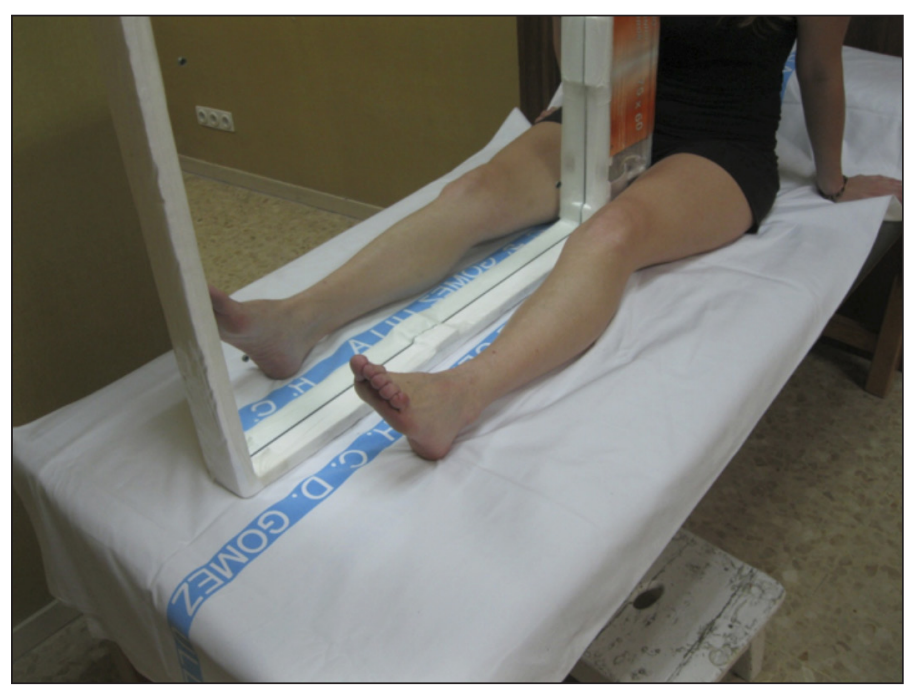

Figura 4. Posicionamiento para el tratamiento del dolor de miembro fantasma mediante la Técnica del Espejo.
La hipótesis es que el cerebro decodifica la nueva información de forma automática, sin que el paciente necesite creerlo, de hecho la persona obviamente sabe que es una ilusión. Ramachandran sugiere que cuando el lóbulo parietal contralateral al miembro amputado experimenta datos conflictivos (como es la incongruencia de señales visuales que le informan al paciente de que su miembro amputado está sano y moviéndose) el cerebro resuelve la incongruencia descartando las señales que proceden del miembro amputado. De esta manera, la huella de memoria del dolor de miembro fantasma es barrida por la nueva información que entra por la vía visual (Figura 5).

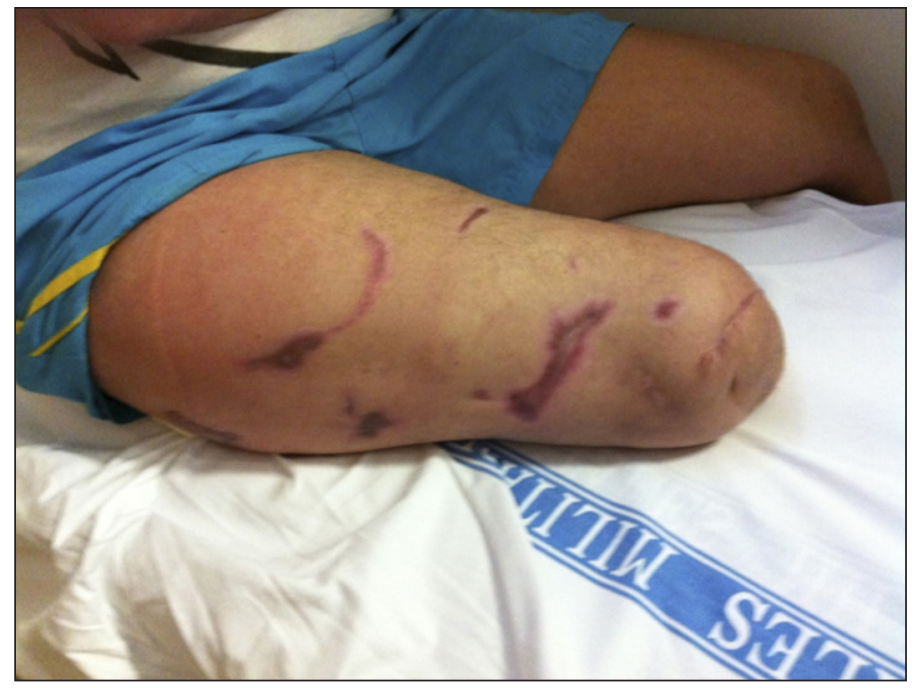

Figura 5. Imagen del miembro afectado en proceso de recuperación.

Según Ramachandran et al. ${ }^{33}$, para que sea realmente efectiva, esta técnica requiere ejercicios de repetición diaria de cinco a diez minutos de duración como mínimo, por espacio de no menos de tres semanas. De esta manera, lo que estaríamos produciendo es un fenómeno de sobre-aprendizaje mediante el cual la información, antigua y dolorosa, sería borrada gradualmente de la memoria del cerebro por la nueva información visual, que indica que el miembro está sano. En términos actuales hablaríamos de una realidad virtual que ofrece una señal de retroalimentación visual externa, que extingue la huella de memoria anterior. Esto pone de manifiesto el sorprendente grado de plasticidad del cerebro adulto, y la poderosa interacción de la vía visual sobre la vía sensitivo-motora, ponderada también por otros autores $3,37,38$.

Se ha informado también del empleo de esta técnica antes de someter a los pacientes a una amputación quirúrgica ${ }^{39}$, encontrando los autores una menor incidencia del desarrollo del DMF tras la amputación. Asimismo, también se ha mostrado efectiva en el tratamiento del dolor de miembro superior posterior a un «stroke».

\section{EVOLUCIÓN DEL CASO}

- Tras la evaluación de la paciente se realizan 30 sesiones como la descrita, de 20-30 minutos en el gimnasio del HCD. Se toma medida subjetiva del DMF al inicio y final 


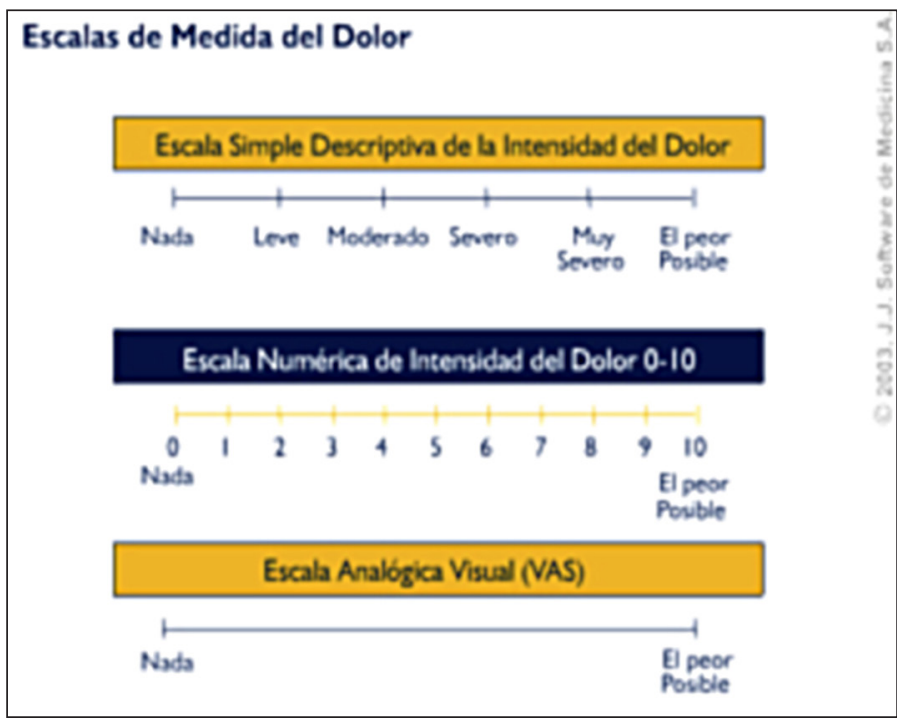

Figura 6. Escala de Valoración Analógica del dolor (EVA).

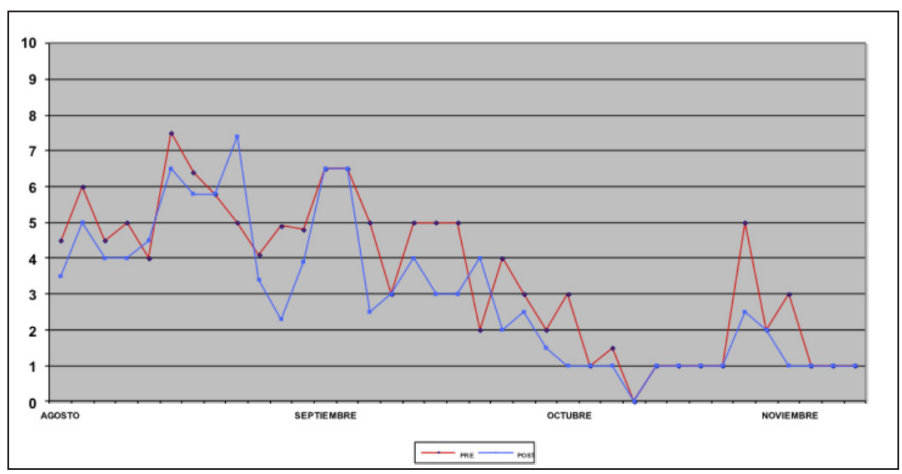

Figura 7. Evolución del DMF en la EVA

de cada sesión mediante EVA (Figura 6) como queda reflejado en la Figura 7. Las EVA constituyen un instrumento habitual para la valoración de la intensidad del dolor $3,37,40$.

- Se produce un incidente digno de reseñar en el que la paciente reexperimenta la pérdida del miembro amputado. Estando en medio de la sesión número 10, contemplando la pierna reflejada en el espejo, sufre episodio de ansiedad en el que rechaza mirar al espejo y refiere no querer creer que tiene la pierna. Por dicho motivo se suspendió el ejercicio hasta el día siguiente.

- Superado este incidente, se continúa con los ejercicios descritos anteriormente y tras 5 sesiones de seguimiento, con niveles de 1 sobre 10, se produce una importante recidiva del dolor. Esta se origina tras haber estado recordando la tarde anterior el atentado con otro compañero, también herido, al que daban de alta. Durante esa noche sufre pesadillas del atentado.
- Se retoman los ejercicios volviendo a alcanzar el nivel de DMF de 1 sobre 10 en tres sesiones.

- Se realizan 4 sesiones más de seguimiento, hasta que la paciente es dada de alta por el Servicio de Rehabilitación. El resultado final pre/post-tratamiento se puede ver en la tabla 1.

\section{CONCLUSIONES}

- El dolor de miembro fantasma es un fenómeno complejo, ampliamente descrito en la literatura científica del que, sin embargo, todavía no tenemos una explicación convincente ni un tratamiento contundente.

- Entre las distintas propuestas sobre el tratamiento de este fenómeno se encuentra la Técnica del Espejo descrita por Ramachandrán. Esta técnica no es efectiva en todos los pacientes, por lo que todavía quedan muchos matices por explicar.

- En el caso descrito, aplicando esta técnica, se muestra como el DMF se reduce tras 4 meses y 30 sesiones de tratamiento, a niveles inferiores a 1, medido en una EVA.

- La aplicación de esta técnica en pacientes que han sufrido amputaciones traumáticas, tienen el inconveniente de la posible reexperimentación del trauma a través de la imagen especular que simula al miembro amputado. Asimismo, también se ha constatado la influencia de aspectos emocionales que pueden actuar como factores moduladores de la experiencia dolorosa y que pueden facilitar o dificultar la adherencia al tratamiento neuropsicológico. En el caso descrito, los estados emocionales de ansiedad provocaron un aumento en la percepción dolor.

- Entre las limitaciones de este trabajo se encuentra el hecho de no haberse podido evaluar ni la personalidad ni el estado de ánimo de la paciente de manera objetiva, así como tampoco la intensidad de los episodios de ansiedad descritos.

- Lo que sí queda de manifiesto es el sorprendente grado de plasticidad del cerebro adulto, y la poderosa interacción de la vía visual sobre la vía sensitivo-motora, como anteriormente han señalado otros autores.

- Por último, necesitamos seguir investigando en el ámbito de la asistencia sanitaria militar operativa para poder ofrecer tratamientos eficaces en este tipo de patologías, tan frecuentes en los escenarios bélicos actuales.

\section{BIBLIOGRAFIA}

1. Gilbert R, González MD. Dolor en los miembros fantasma y dolor de muñón. Rev. Soc. Esp. Dolor 1996; vol. 3: 283-289.

Tabla 1. Resultado prelpost-tratamiento de la descripción del dolor.

\begin{tabular}{|lll|}
\hline \multicolumn{1}{|c|}{ DESCRIPCIÓN } & \multicolumn{1}{c|}{ PRETRATAMIENTO } & PVSTRATRAMIENTO \\
\hline Intensidad & EVA 5-6 (0-10) & E-10) \\
Cualidad & Como una corriente eléctrica & Agarrotamiento \\
Sensación & Espasmo & Adormecimiento, hormigueo \\
Localización & En el puente del pié & $i ?$ \\
Vivencia & Agobiante, angustioso, desesperante, constante & Momentáneo, leve \\
\hline
\end{tabular}


2. Vaquerizo A. Dolor postamputación. Rev. Soc. Esp. Dolor 2000; Vol. 7, suplemento II: pp. 60-77.

3. Wilcher G, Chernev I, Yan K. Combined mirror visual and auditory feedback therapy for upper limb phantom pain: a case report. J. Medical Case Reports 2011; 5;41.

4. Sherman RA, Sherman CJ. A comparison of phantom sensations among amputees whose amputations were pf civilian and military origins. Pain 1985; 21: 91-97.

5. Kessel C, Wörz R. Immediate response of phantom limb pain to calcitonin. Pain 1987; 30: 79-87.

6. Melzack R. Phantom limbs. Sci. Am. 1992; 226: 90-96.

7. Bartusch SL, Sanders BJ, D'Alessio JG, Jernigan JR. Clonazepam for the treatment of lancinating phantom limb pain. Clin. J. Pain 1996; 12: 59-62.

8. Montoya P, Larbig W, Grulke N, Flor H, Taub E. The relationship of phantom limb pain to other phantom limb phenomena in upper extremity amputees. Pain 1997; 72: 87-93.

9. Flor H, Birbaumer N, Sherman RA. Phantom limb pain. Rev. Soc. Esp. Dolor 2001. 8: 327-331.

10. Davis RW. Phantom sensations, phantom pain, and stump pain. Arch. Phys. Med. Rehabil. 1993; 74: 79-91.

11. Katz J. Psychophysiological contributions to phantom limbs. Can. J. Psychiatry 1992; 37: 282-298.

12. Sherman R, Sherman CJ, Gall N. A survey of current phantom limb pain treatment in the United States. Pain 1980; 8: 85-99.

13. De Ridder D, Elgoyhen AB, Romo R, Langguth B. Phantom percepts: Tinnitus and pain as persisiting aversive memory networks. Natl. Acad. Sci. USA 2011; 108-20: 8075-8080.

14. Sherman RA, Sherman CJ, Bruno GM. Psychological factors influencing chronic phantom limb pain: an analysis of the literature. Pain 1987; 285-295.

15. Ramachandran VS, Hirstein W. The perception of phantom limbs. Brain 1998:121:1603-1630

16. Nikolajsen, L. Phantom limb pain, en Evidence-Based Chronic Pain Management. Edited by C. Stannard, E. Kalso and J. Ballantyne. 2010 Blackwell Publishing. Cap. 19, pp 237- 247

17. Foell J, Bekrater-Bodmann R, Flor H, Cole J. Phantom Limb Pain After Lower Limb Trauma: Origins and Treatments. Int. J. Low Extrem Wounds 2011; 10-4: 224-235.

18. Flor $\mathrm{H}$, Nikolajsen $\mathrm{L}$, Jensen TS. Phantom limb pain: a case of maladaptive CNS plasticity? Nature Rev Neurosci 2006; 7: 873-881.

19. Kooijman CM, Dijkstra PU, Geertzen JHB, et al. Phantom pain and phantom sensations in upper limb amputees: an epidemiological study. Pain 2000 87: $33-41$.

20. Nikolajsen L, Ilkjær S, Jensen TS. Relationship between mechanical sensitivity and postamputation pain: a prospective study. Eur J Pain 2000; 4: $327-334$.

21. Flor H, Denke C, Schaefer M, et al. Effect of sensory discrimination training on cortical reorganization and phantom limb pain. Lancet 2001; 357 1763-1764.
22. Aydin MD, Cesur M, Aydin N, Alici HA. Disappearance of phantom limb pain during cauda equina compression by spinal meningioma and gradual reactivation after decompression. Anesth Analg 2005; 101: 1123-1126.

23. Tessler MJ, Kleiman SJ. Spinal anaesthesia for patients with previous lower limb amputations. Anaesthesia 1994; 49:439-441.

24. Nikolajsen L, Hansen CL, Nielsen J, et al. The effect of ketamine on phantom pain: a central neuropathic disorder maintained by peripheral input. Pain 1996; 67: 69-77.

25. Florence SL, Kaas JH. Large-scale reorganization at multiple levels of the somatosensory pathway follows therapeutic amputation of the hand in monkeys. J Neurosci 1995; 15:8083-8095.

26. Pons TP, Garraghty PE, Ommaya AK, et al. Massive cortical reorganization after sensory deafferentation in adult macaques. Science 1991; 252: 1857-1860.

27. Flor H, Elbert T, Knecht S. Phantom limb pain as a perceptual correlate of cortical reorganization following arm amputation. Nature 1995; 375: 482-484.

28. Flor H, Elbert T, Mühlnickel W. Cortical reorganization and phantom phenomena in congenital and traumatic upperextremity amputees. Exper Brain Res 1998; 119: 205-212.

29. Birbaumer N, Lutzenberger W, Montoya P, et al. Effects of regional anesthesia on phantom limb are mirrored in changes in cortical reorganization in upper limb amputees. J Neurosci 1997; 17: 5503-5508.

30. Huse E, Larbig W, Flor H, et al. The effect of opioids on phantom limb pain and cortical reorganization. Pain 2001; 90: 47-55.

31. Davis KD, Kiss ZH, Luo L. Phantom sensations generated by thalamic microstimulation. Nature 1998; 391: 385-387.

32. Draganski B, Moser T, Lummel N, et al. Decrease of thalamic gray matter following limb amputation. NeuroImage 2006; 31: 951-957.

33. RamachandranVS. Rogers-Ramachandran D, Teward M. Perceptual Correlates of Massive Cortical Reorganization. Science 1992; 258, 1159-60.

34. Alviar MJM, Hale T, Dungca M. Pharmacologic interventions for treating phantom limb pain. Cochrane Database of Systematic Reviews 2011, Issue 12. Art. No.: CD006380. DOI: 10.1002/14651858. CD006380.pub2.

35. Solvey P, Ferrazzano RC. Miembro fantasma. En $6^{\circ}$ Congreso Virtual de Psiquiatría. Interpsiquis 2005 . Psiquiatria.com

36. Ramachandran VS, Rogers-Ramachandran D. Synaesthesia in phantom limbs induced with mirrors. Proc. Biol. Sci. 1996; 263:377-386.

37. Mercier C, Sirigu A. Training With Virtual Visual Feedback to Alleviate Phantom Limb Pain. Neurorehabil. Neural Repair 2009; 23-6: 587-594.

38. Nojima I, Mima T, Koganemaru S, Nasreldin M, Fukuyama H, Kawamata T. Human Motor Plasticity Induced by Mirror Visual Feedback. J. Neurosc. 2012; 32-4: 1293-1300.

39. Hanling SR, Wallace SC, Hollenbeck KJ, Belnap BD, Tulis MR. Preamputation Mirror Therapy May Prevent Development of Phantom Limb Pain: A case series. Anesth. Analg. 2010; 110-2: 611-614

40. Cacchio A, De Blasis E, De Blasis V, Santilli V, Spacca, G. Mirror Therapy in Complex Regional Pain Syndrome Type 1 of the Upper Limb in Stroke Patients. Neurorehabil. Neural Repair 2009; 23-8: 792-799. 